\title{
Los cambios de hábitos de vida y la metformina mantuvieron su eficacia en la prevención de diabetes a largo plazo
}

\author{
Life style changes and metformin conserved their long term efficacy to prevent diabetes
}

Diabetes Prevention Program Research Group. Lancet. 2009;374:1677

\section{Introduccion}

El Programa para la Prevención de Diabetes (PPD) fue un ensayo clínico multicéntrico aleatorizado doble ciego, realizado en EE.UU entre 1999 y 2001, y que había demostrado la reducción de la incidencia de diabetes a mediano plazo en una población adulta de alto riesgo, a través intervenciones intensivas para promover cambios de hábitos de vida, así como con metformina.

\section{Objetivo}

Determinar si el retraso en el desarrollo de diabetes que se observó a mediano plazo se mantiene a los diez años.

\section{Diseño, lugar y pacientes}

Ensayo clinico aleatorizado controlado llevado a cabo en 27 centros de EE.UU. Participó el $88 \%(n=2766)$ de la población original del PPD, cuyos criterios de inclusión habían sido tener una glucemia alterada en ayunas o intolerancia a la glucosa y un índice de masa corporal de $24 \mathrm{~kg} / \mathrm{m}^{2}$ ó mayor. La edad promedio fue de 50 años y el $68 \%$ fue de sexo femenino.

\section{Intervención y medición de resultados principales}

Al finalizar el estudio original se ofreció a todos los participantes un programa educativo sobre cambios de hábitos de vida (concurrencia de $57 \%$ del grupo "placebo" original, $58 \%$ del grupo "metformina" y $40 \%$ del grupo "cambios de hábito"). Los pacientes de la rama original de metformina continuaron recibiéndola en una dosis de $850 \mathrm{mg}$, dos veces por dia.
Se consignó el desarrollo de diabetes según los criterios de la Asociacion Americana de Diabetes (ADA) y el análisis fue realizado por intención de tratar.

\section{Resultados Principales}

La incidencia de diabetes durante el seguimiento inicial del DPP había sido de 4,8 casos por cada 100 personas/año (IC95\% 4,1 a 5,7$)$ en el grupo de cambios de hábito, $7,8(6,8$ a 8,8$)$ en el grupo metformina y $11,0(9,8$ a 12,3) en el grupo placebo. La incidencia de diabetes en este estudio de seguimiento fue similar en los tres grupos: $5,9(5,1$ a 6,8$) ; 4,9(4,2$ a 5,7$)$ y $5,6(4,8$ a 6,5$)$ respectivamente. Tomando como referencia al grupo placebo, la incidencia de diabetes durante los diez años desde la aleatorización fue un 34\% (IC95\% 24 a 42) menor en el grupo asignado a cambios de hábitos y un $18 \%$ (IC95\% 7 a 28) en el grupo metformina.

\section{Conclusiones}

La incidencia acumulada de diabetes fue menor en el grupo que realizó cambios en el estilo de vida. La prevención del desarrollo de diabetes o el retraso de su aparición producidos por los cambios del estilo de vida y/o por la metformina, persistieron al menos diez años.

Palabras claves: incidencia de diabetes, cambios de hábitos de vida, metformina, Palabras claves: incidencia

Keywords: Diabetes incidence, llifestyle habits, metformin, long-term follow-up. Fuentes de financiamiento: National Institute of Diabetes and Digestive and Kidney Diseases.

\section{Comentario}

Es interesante conocer algunos detalles del programa que se utilizó en el PPD para promover cambios de hábitos de vida con el objetivo de ayudar a los pacientes a conseguir un descenso de peso del 7\% y a realizar 150 minutos o más de actividad física moderada por semana. Los pacientes elegían en que focalizar primero. Se les entregó un manual para completar las recomendaciones en forma personalizada -cuantos kilos tendría que bajar, que actividad física realizaría, cuando, donde y con quien, etc.- haciendo hincapié en cambios graduales, saludables y coherentes con la vida cotidiana de cada paciente. Por ejemplo, incorporando actividad física durante las siguientes dos semanas y monitoreando lo que se comería. Paso a paso, iban progresando en la actividad física con ejercicios específicos para algunos músculos, hasta realizar un plan de actividades. En relación a la dieta, primero se los entrenó a detectar grasas en las comidas y modificar las formas de preparación, hasta llegar a una dieta saludable. También se trabajó sobre como lograr un habitat saludable, sobre cómo resolver problemas y sobre como mantener los cambios alcanzados. Por último, y al finalizar el curso se les entregó otro manual'1. Otro aspecto importante a destacar en este estudio es que demostró que en estos pacientes también la metformina disminuye la incidencia de diabetes, aunque menos que los cambios de hábito. Vale destacar que hasta hace poco no existía evidencia clara que avalara el tratamiento farmacologico en estos pacientes ${ }^{2}$, y que junto con la consejería sobre cambios de hábitos para prevenir el desarrollo de diabetes, la ADA actualmente recomienda el tratamiento con metformina en pacientes de alto riesgo definidos por intolerancia a la glucosa o glucemia alterada en ayunas más alguno de los siguientes criterios: hipertensión arterial, colesterol HDL bajo, triglicéridos elevados, historia de diabetes en familiar de primer grado, obesidad y edad menor a 60 años, o historia de diabetes gestacional.

\section{Conclusiones de la comentadora}

Con el seguimiento a largo plazo, la estrategia cambio de hábitos siguió siendo la más beneficiosa. A pesar de su difícil aplicación, es importante su implementación, ya sea sola o asociada a la metformina.

Carolina Begue [ Servicio de Medicina Familiar y Comunitaria del Hospital Italiano de Buenos Aires. Carolina.begue@hospitalitaliano.org.ar ]

Begue C. Los cambios de hábitos de vida y la metformina mantuvieron su eficacia en la reducción de diabetes a largo plazo. Evid. Actual. Práct. Ambul; 13(1): 7, Ene-Mar 2010. Comentado de: Diabetes Prevention Program Research Group. Lancet. 2009;374:1677-86. PMID: 19878986. Disponible en URL: http://www.thelancet.com/journals/lancet/article/PIIS0140-6736\%2809\%2961457-4/fulltext

Referencias

1. http://www.bsc.gwu.edu/dpp/index.htmlvdoc

2. Gillies $\mathrm{C}$ y col. Pharmacological and lifestyle interventions to prevent or delay type 2 diabetes in people with impaired glucose tolerance: systematic review and meta-analysis. BMJ. 2007;334(7588):299 